\title{
Assessment of directional doppler ultrasound techniques in the diagnosis of carotid artery diseases
}

\author{
D. R. P R I C H A R D, T.R. P. M A R T IN, A N D S US A N B. SHER I F F \\ From Sheffield University and Area Health Authority (Teaching), Sub-Regional Department of \\ Medical Physics and Department of Neurology, Fulwood Annexe, Sheffield
}

SUMMARY Three diagnostic techniques based on the use of directional doppler ultrasound have been evaluated in comparison with direct percutaneous carotid angiography and arch aortography. The results indicate that the non-invasive techniques provide a safe, atraumatic method of assessing patients with symptoms of extracranial occlusive arterial disease.

The identification of occlusive carotid artery disease is often critical in the management of supratentorial stroke. Specific examination of the major blood vessels in the neck by palpation and auscultation may help in reaching a tentative diagnosis. However, the demonstration of a lesion in the common carotid artery and its branches will inevitably depend on the use of contrast radiography.

According to Deck (1976) and Hass et al. (1963) major complications may follow angiography in $0.5-1 \%$ of cases with minor sequelae in up to $14 \%$. Further, the incidence of such complications rises with age. This has deterred many physicians from carrying out investigations requiring arterial puncture. In addition the incidence of operable carotid stenosis in patients presenting with transient ischaemic attacks is low-Bradshaw and Gumpert (1972) found occlusive carotid disease in only 19 of 69 patients, and in only nine of these was there operable stenosis.

The aetiology of transient cerebral ischaemia is complex. For these reasons the development of non-invasive techniques that can detect lesions in the vessels supplying the affected cerebral hemisphere has been pursued. These include ophthalmodynamometry (Liversedge and Smith, 1961), thermography (Wood, 1965), carotid phonoangiography (Duncan et al., 1975), ocular plethysmography (Gee et al., 1974), and radioisotope cerebral perfusion (Weissman et al., 1975).

Diagnostic techniques based on directional doppler ultrasound have been reported (Brocken-

Address for reprint requests: Dr T. R. P. Martin, Sub-Regional Department of Medical Physics, Hallamshire Hospital, Sheffield S10 2JF.

Accepted 21 December 1978 brough, 1970; Muller, 1972; Machleder, 1973; Nuzzaci et al., 1975; Bone and Barnes, 1976a; Keller et al., 1976; Baskett et al., 1977; Rutherford et al., 1977). Potentially they provide a safe, atraumatic procedure for the assessment of patients with transient ischaemic attacks. In the present paper we present the results of an 18 month study in which techniques based on the use of directional doppler ultrasound were evaluated in comparison with direct percutaneous carotid angiography and arch aortography.

\section{Subjects and methods}

The patients studied were those referred for the investigation of transient ischaemic attacks in the carotid artery territory and who subsequently underwent angiographic investigation.

A tracing of the vessel outline based on the anterior-posterior view of the angiogram was used to classify each vessel into one of five groups (Table 1). The normal group represents those vessels as shown by the radiograph to be completely free from occlusive disease. Tortuosity was accepted as normal if no occlusive disease was

Table 1 Vessel segments classified according to arteriographic findings in 60 patients

\begin{tabular}{ll}
\hline Classification & Number of vessel segments \\
\hline Normal & 25 \\
Minimal atheroma & 10 \\
Stenosis & 18 \\
Less than $75 \%$ & 7 \\
Greater than $75 \%$ & 16 \\
Occlusions & 76 \\
$\quad$ Total & \\
\hline
\end{tabular}


present. The minimal atheroma classification includes those vessels with irregularities of the wall without measurable loss in lumen diameter. The classification of stenosis was based on the reduction in vessel lumen from the projected vessel diameter at the point of maximum stenosis. Occlusions refer to complete obstruction of the internal carotid or common carotid arteries.

The patient was placed in a supine position in a warm room $\left(25 \pm 2^{\circ} \mathrm{C}\right)$. After an initial 15 minute stabilisation period the brachial blood pressure was taken in both arms using a sphygmomanometer. A $10 \mathrm{MHz}$ ultrasonic probe was placed below the level of the thyroid cartilage medial to the sternocleidomastoid muscle in the lower apex of the anatomical carotid triangle. The probe was inclined so that the ultrasound beam was directed downwards towards the root of the neck. Doppler shift signals were processed using a Parks 80610 $\mathrm{MHz}$ Directional Doppler in conjunction with a real time frequency analyser (Smallwood and Brown, 1977). The frequency analysed doppler signals were displayed on a storage oscilloscope. The probe position was adjusted to give the clearest and most representative signal on the screen free from artefacts caused by vessel wall movement and venous flow. The frequency analysed doppler shift signals were then recorded on a fibre optic recorder (Medelec FOR-7) at $50 \mathrm{~mm}$ $\mathrm{s}^{-1}$, to give a tracing as shown in Fig. 1a. The density of the trace is an indication of the relative amount of blood with a given velocity.

This procedure was repeated with the probe placed in the upper medial angle of the orbit with the beam directed towards the supratrochlear artery as it emerges from the orbit. Great care was taken to ensure that the ultrasound beam was not directed towards the sensitive structures lying within the eyeball. In most cases the direction of flow could be identified clearly as physiological, that is flow towards the probe, or pathological, flow away from the probe (Muller, 1972) (Fig. 1c and 1d). However, in a small number of cases the directional information was ambiguous with both forward and reverse flow components present. In such cases no amount of probe reorientation could resolve a direction.

The common carotid and supratrochlear signals have a characteristic wave shape from which two well-defined peaks can usually be distinguished. The relationship between these two peaks appears to be modified in the presence of occlusive disease (Fig. 1b). The extent of this change can be quantified by taking the ratio of the first peak over the secrind (A/B ratio) as defined by Gosling (1976). This ratio was calculated from the fre-

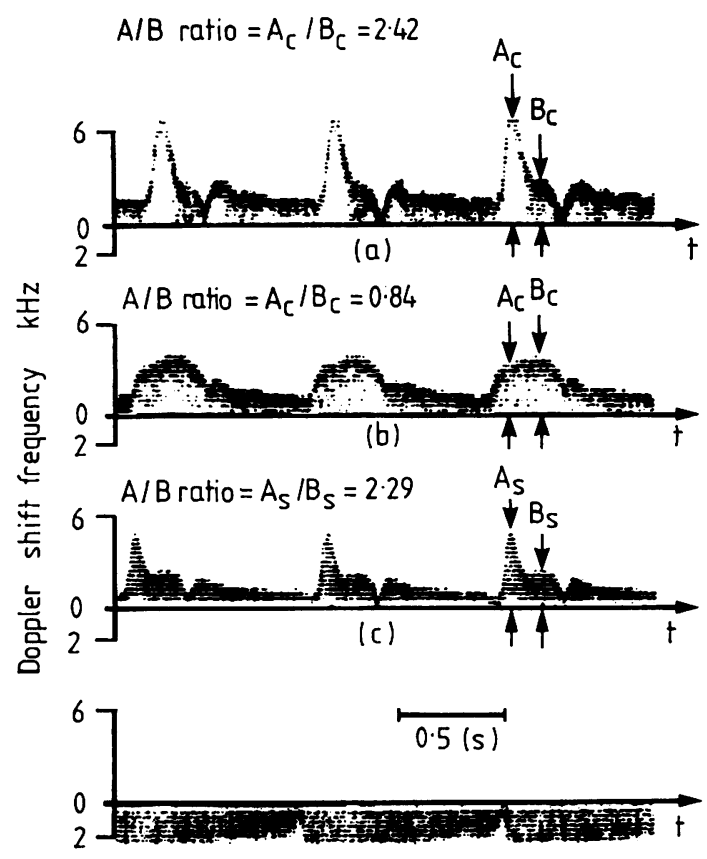

(d)

Fig. 1 Frequency analysed doppler shifted signals recorded from: (a) the common carotid artery in a patient with a normal bifurcation and extracranial vessels (normal group); (b) the common carotid artery in a patient with internal carotid disease (less than $75 \%$ stenosis group); (c) the supratrochlear artery in a patient with a normal bifurcation and extracranial vessels (normal group); (d) the supratrochlear artery in a patient with severe occlusive disease of the internal carotid showing pathological reverse flow (greater than $75 \%$ stenosis group).

quency analysed doppler shifted records obtained from both the common carotid and supratrochlear arteries.

The main branches of the external carotid artery that anastomose with the internal carotid via the ophthalmic artery are the superficial temporal, facial, and infraorbital arteries (Fig. 2). The effect of compressing each of these vessels in turn, both homolateral and contralateral, on the magnitude and direction of the supratrochlear doppler signal was recorded on 67 of the 76 vessels studied.

\section{Results}

SUPRATROCHLEAR FLOW

In Table 2 the direction of flow in the supratrochlear artery is related to the arteriographic findings. The results of compressing the external 


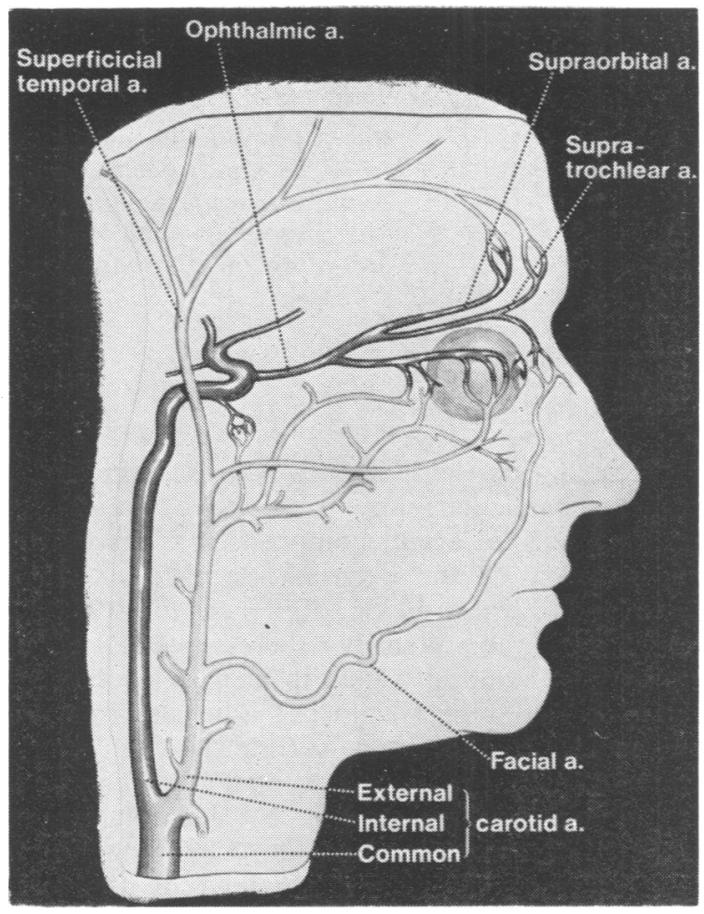

Fig. 2 The common carotid artery and its principal branches.

Table 2 Direction of flow in the supratrochlear artery related to arteriographic findings

\begin{tabular}{lrlll}
\hline Classification & \multicolumn{2}{c}{ Direction of flow } & $\begin{array}{l}\text { Total number } \\
\text { of vessels }\end{array}$ \\
\cline { 2 - 4 } & Forward & Reverse & Ambiguous & \\
\hline Normal & 25 & 0 & 0 & 25 \\
Minimal atheroma & 9 & 0 & 1 & 10 \\
Stenosis & 15 & 1 & 2 & 18 \\
$\quad$ Less than 75\% & 0 & 7 & 0 & 7 \\
$\quad$ Greater than 75\% & 4 & 9 & 3 & 16 \\
Occlusion & 53 & 17 & 6 & 76 \\
Total & & & & \\
\hline
\end{tabular}

carotid branches on the direction of flow in the supratrochlear vessels are given in Table 3. The vessel responses are defined as follows: a normal response is the enhancement of flow in the supratrochlear vessel on compression of one of the previously stated branches of the external carotid; a negative response is no response at all to compression of any of these vessels whether homolateral or contralateral; a positive response is a reduction in flow, on compressing one of the vessels.

ANALYSIS OF WAVE SHAPE

The A/B ratios calculated from the supratrochlear and common carotid arteries for each vessel segment have been plotted according to arteriographic grouping (Fig. 3). Vessel segments with reverse flow in the supratrochlear artery have not been plotted; neither have the four occlusions with forward flow and the vessel segments with ambiguous signals from the supratrochlear artery.

\section{Discussion}

Reverse flow as an indication of occlusion is only found in $56 \%$ of cases (Table 2). This is in general agreement with the results of Gado and Marshall (1971) who found $76 \%$ of cases to have reversed flow by angiography. These results are contrary to the findings of Brockenbrough (1970), Baskett et al. (1977), and Bone and Barnes (1976a and b) who indicated reverse flow obtaining in all cases of occlusion. In the cases of forward flow and angiographic evidence of occlusion the supply must be from either the contralateral internal carotid or the vertebral system via the circle of Willis. The finding of forward flow is, therefore, possible in an occlusion but its source will differ from the forward flow found in the normal, physiological state and in cases of stenosis.

Table 3 Results of compression tests while monitoring the supratrochlear signal. Compression studies were not done in nine cases. Ambiguous signals were obtained in six cases which are not included in the table. Of these, four gave a normal response, one a negative, and one a positive response

\begin{tabular}{|c|c|c|c|c|c|c|c|}
\hline \multirow[t]{3}{*}{ Classification } & \multicolumn{6}{|c|}{ Direction of flow } & \multirow[t]{3}{*}{ Total number of vessels } \\
\hline & \multicolumn{3}{|l|}{ Forward } & \multicolumn{3}{|l|}{ Reverse } & \\
\hline & Normal & Negative & Positive & Normal & Negative & Positive & \\
\hline Normal & 16 & 8 & 0 & 0 & 0 & 0 & 24 \\
\hline Minimal atheroma & 5 & 2 & 0 & 0 & 0 & 0 & 7 \\
\hline \multicolumn{8}{|l|}{ Stenosis } \\
\hline Less than $75 \%$ & 7 & 3 & 0 & 1 & 0 & 1 & $11^{*}$ \\
\hline Greater than $75 \%$ & 0 & 0 & $\mathbf{0}$ & 1 & 2 & 5 & $7 *$ \\
\hline Occlusions & 3 & 1 & 0 & 1 & 1 & 7 & $12 *$ \\
\hline
\end{tabular}

\footnotetext{
*In these cases two responses were obtained depending on which vessel was compressed.
} 


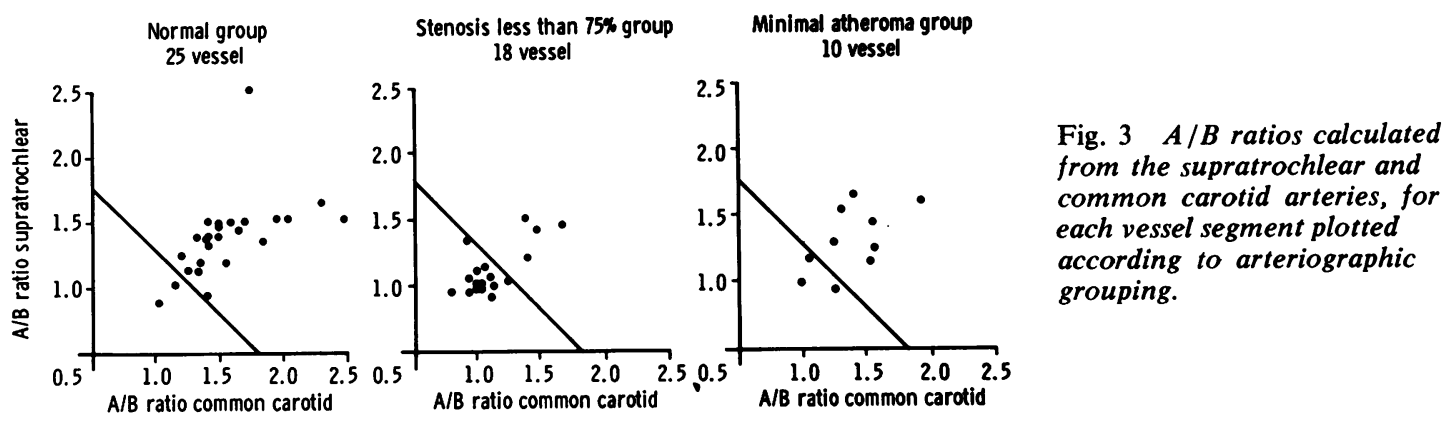

Reverse flow is always pathological. As mentioned above it was found in $56 \%$ of all occlusions. It was also found in $23 \%$ of stenoses. Only those stenoses severe enough to cause a considerable distal drop in pressure will give reversal of flow. Deweese et al. (1970) have suggested that a reduction in diameter of $63 \%$ or a loss of luminal area of $87 \%$ is necessary for flow reversal to occur. Other authors (Bone and Barnes, 1976a) have taken $75 \%$ as the figure for the reduction in diameter, which is in agreement with the results presented in this paper.

The occurrence of forward flow in the ophthalmic branches originating from vessels other than the homolateral common carotid was only seen in longstanding internal carotid occlusion. None of the severe stenosis group (greater than $75 \%$ reduction in diameter) was found to have forward flow. This may indicate that the development of collateral pathways occurs once reverse flow has been established.

It has been suggested that by the use of compression of the external carotid branches further information can be obtained. However, as shown in Table 3 the amount of information that we have found valuable using this procedure is limited, since compression tests did not separate the groups with forward flow. The results do show that a positive response is pathological. Three cases of reverse flow gave both a normal and a positive response depending on which branch of the external carotid was compressed. This suggests that there is a balance of flow not only between the internal and external carotid arteries but also between branches of the external carotid in those patients.

In summary, the direction of flow in the orbit and compression of the external carotid branches do not help to identify the following groups that are important: (1) occlusions with forward flow; (2) stenosis with reverse flow from occlusions with reverse flow; (3) areas of atheroma and stenosis less than $75 \%$ of vessel diameter.
On reviewing the literature it is clear that many authors, particularly overseas, have found that common carotid artery compression is a vital and routine part of the examination (Muller, 1972; Bone and Barnes, 1976b; Keller et al., 1976). It would certainly identify those categorised in group 1. It could also help in identifying those in group 2. Although we do not advocate this procedure, it is clear that certain lesions can be missed if examined by directional ultrasound and limited compression studies. No information regarding lesions causing less than $75 \%$ reduction in vessel diameter has been obtained by the technique discussed above. A simple form of wave $\stackrel{\mathbb{D}}{\circ}$ shape analysis of the common carotid and supra-O trochlear frequency analysed doppler shift signals $\triangle$ reveals a difference between normal and diseased vessels (Fig. 3). The results compare favourably with the work of Baskett et al. (1977). The dividing line shown in Fig. 3 indicates that by the addition of the two $A / B$ ratios obtained from the common carotid and supratrochlear vessels the information is reduced to a single parameter which separates the three groups (Fig. 4).

The aim of the study has been to assess the application of non-invasive directional continuous wave doppler ultrasound techniques in the diagnosis of disease in the common and internal carotid arteries. We have shown that compression of the external carotid branches is only of value in identifying the source of reverse flow. Combining the techniques of direction of flow in the supratrochlear vessels and waveform analysis of the common carotid and supratrochlear signals, the results of this form of examination when compared with arteriographic findings are shown in Fig. 5. This shows that the non-invasive techniques discussed above provide a safe, atraumatic method of assessing patients with symptoms of extracranial arterial disease. The tests are most accurate when examining patients with severe disease of the carotid vessels. 


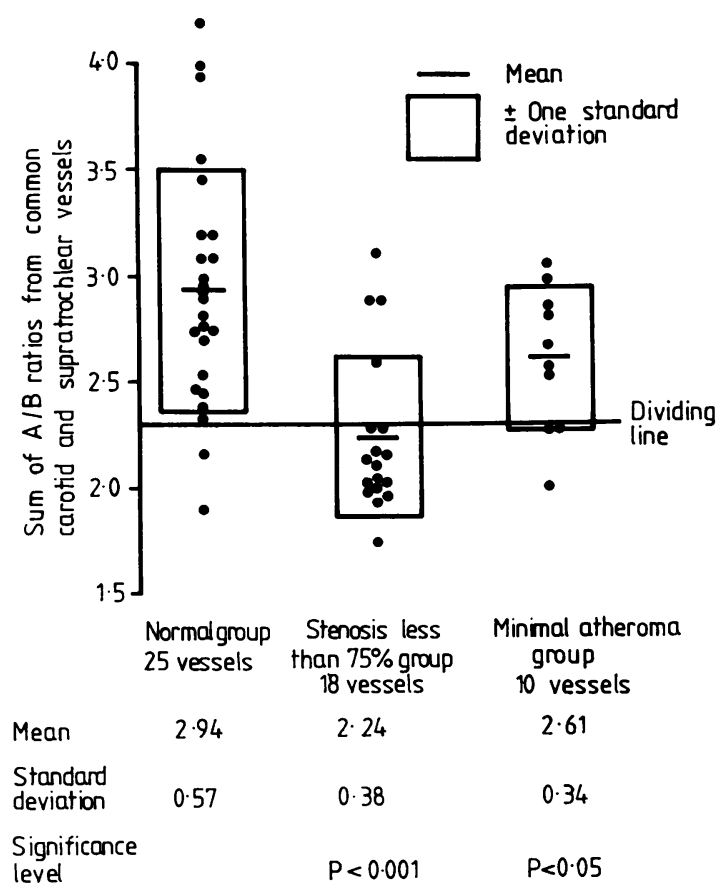

(Student's $t$ test)

Fig. 4 Sum of $A / B$ ratios calculated from the supratrochlear and common carotid arteries for each vessel segment plotted according to arteriographic grouping. The mean and standard deviation for each group are shown.

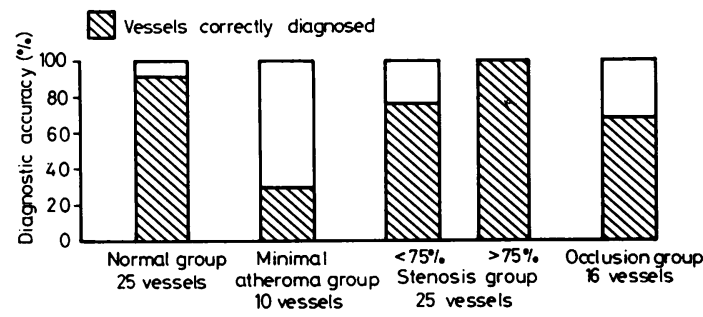

Fig. 5 Accuracy of doppler ultrasound based techniques in diagnosing normal and diseased vessels when compared with angiography.

We would like to express our thanks to Dr J. P. P. Bradshaw, Dr G. A. B. Davies-Jones, and Dr J. Gumpert for their permission to examine patients admitted under their care. The support of the consultant radiologists in the Sheffield area is also gratefully acknowledged. Financial support was provided by the Ryder Briggs Research Fellowship.

\section{References}

Baskett, J. J., Beasley, M. G., Murphy, G. J., Hyams, D. E., and Gosling, R. G. (1977). Screening for carotid junction disease by spectral analysis of doppler signals. Cardiovascular Research, 11, 147155.

Bone, G. E., and Barnes, R. W. (1976a). Clinical implications of the doppler cerebrovascular examination: a correlation with angiography. Stroke, 7, 271-274.

Bone, G. E., and Barnes, R. W. (1976b). Limitations of the doppler cerebrovascular examination in hemispheric cerebral ischaemia. Surgery, 79, 577580.

Bradshaw, P., and Gumpert, J. (1972). Small strokes in the carotid territory associated with a normal carotid arteriogram. Journal of Neurology, Neurosurgery, and Psychiatry, 35, 810-817.

Brockenbrough, E. G. (1970). Screening for the prevention of stroke: use of a doppler flow meter. Washington/Alaska, Regional Medical Program Brochure.

Deck, M. D. F. (1976). Radiologic examination of patients with stroke. Postgraduate Medicine, 59, 123-131.

Deweese, J. A., May, A. G., Lipechik, E. O., and Rob, C. G. (1970). Anatomic and hemodynamic correlations in carotid artery stenosis. Stroke, 1, 149-157.

Duncan, G. W., Grubec, J. O., and Dewey, C. F. (1975). Evaluation of carotid stenosis by phonoangiography. New England Journal of Medicine, 293, 1124-1128.

Gado, M., and Marshall, J. (1971). Clinico-radiological study of collateral circulation after internal carotid and middle cerebral occlusion. Journal of Neurology, Neurosurgery, and Psychiatry, 34, 163-170.

Gee, W., Smith, C. A., Hinson C. E., and Nylie, E. J. (1974). Ocular pneumoplethysmography in carotid artery disease. Medical Instrumentation, 8, 244248.

Gosling, R. G. (1976). Extraction of physiological information from spectrum analysed doppler shifted continuous wave ultrasound signals obtained noninvasively from the arterial system. In IEE Medical Electronics Monographs, vols. 13-22, pp. 33-125. Edited by D. W. Hill and B. W. Watson. Peter Peregrinus: Stevenage, Hertfordshire.

Hass, W. K., Fields, W. S., North, R. R., Kricheff, I. I., Chase, N. E., and Bauer, R. B. (1963). Joint study of extracranial arterial occlusion. Journal of the American Medical Association, 203, 159-166.

Keller, H., Meier, W., Yonekawa, Y., and Kumpe, D. (1976). Non-invasive angiography for the diagnosis of carotid artery disease using doppler ultrasound. Stroke, 7, 354-363.

Liversedge, L. A., and Smith, V. H. (1961). The place of ophthalmodynamometry in the investigation of cerebrovascular disease. Brain, 84, 274-288.

Machleder, H. I. (1973). Evaluation of patients with cerebrovascular disease using the doppler ophthalmic 
test. Proceedings of the 19th Annual Meeting of the American College of Angiology, San Juan, Puerto Rico.

Muller, H. R. (1972). The diagnosis of internal carotid occlusion by directional doppler sonography of the ophthalmic artery. Neurology, 22, 816-823.

Nuzzaci, G., Briani, S., Mennomma, P., and Evangelist, A. (1975). The doppler ophthalmic test. Neurosurgical Sciences, 19, 129-138.

Rutherford, R. B., Hiatt, W. R., and Kreutzer, E. W. (1977). The use of velocity wave form analysis in the diagnosis of carotid artery occlusive disease. Surgery, 82, 695-702.

Smallwood, R. H., and Brown, B. H. (1977). A real time frequency analyser for ultrasonic signals. Jour nal of Medical Engineering and Technology, 1, 221-222.

Weissman, B. N., Holman, B. L., and Rosenbaum, A. E. (1975). Radionuclide angiography in graded carotid stenosis. Radiology, 115, 399-402.

Wood, E. H. (1965). Thermography in the diagnosis of cerebrovascular disease. Radiology, 85, 270-283. 